\title{
Precision of Independently Based Gain and Offset Error of an ADC using the Histogram Method
}

\author{
F. Corrêa Alegria ${ }^{(1)}$, Member, IEEE, A. Cruz Serra ${ }^{(2)}$, Senior Member, IEEE \\ (1) Instituto de Telecomunicações / Instituto Superior Técnico, Technical University of Lisbon, Av. Rovisco Pais, 1049-001 \\ Lisboa, Portugal, Ph: +351-218418376 Fax: +351-218417672, E-mail: falegria@lx.it.pt, \\ (2) Instituto de Telecomunicações / Instituto Superior Técnico, Technical University of Lisbon, Av. Rovisco Pais, 1049-001 \\ Lisboa, Portugal, Ph: +351-218418490 Fax: +351-218417672, E-mail: acserra@ist.utl.pt
}

\begin{abstract}
Two of the parameters that are determined when testing an analog to digital converter are the gain and offset error. One of the ways to define these two parameters is called "Independently Based". Here we derive the precision of the gain and offset error estimated with the Histogram Test Method affected by additive noise.
\end{abstract}

Index Terms-ADC, Gain, Offset Error, Histogram, Precision.

\section{INTRODUCTION}

The transfer function of an analog to digital converter (ADC) relates the analog input voltage (or current) to the digital output code (Fig. 1). In the following we will consider that the ADC input is a voltage. The number of output codes depends on the ADC resolution $\left(n_{b}\right)$ and is equal to $2^{n_{b}}$. The transition voltage $T_{k}$ is, by definition, the value of constant input voltage that leads to an equal amount of output codes lower than $k$ and equal or higher than $k$. Different output codes are expected for a constant input voltage due to the inevitable presence of noise. The code bin width, $W_{k}$, is the distance between the two consecutive transition voltages $T_{k}$ and $T_{k+1}$.

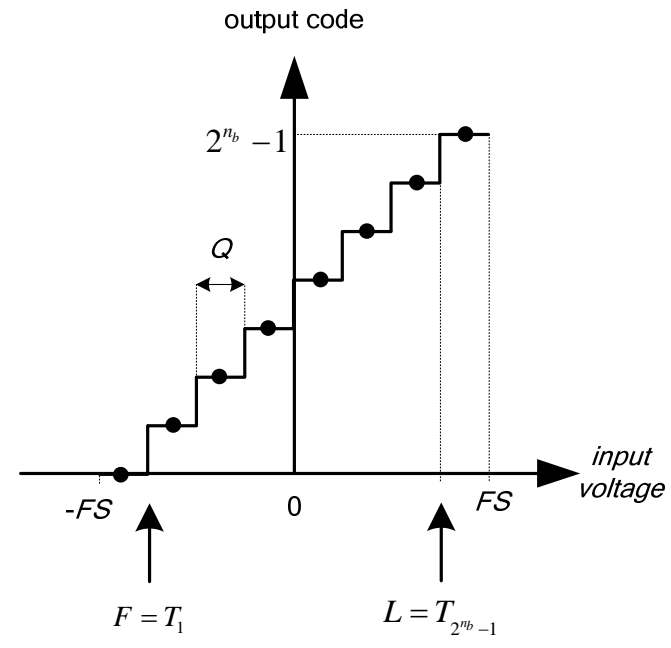

Fig. 1 - Illustration of the transfer function of a symmetrical bipolar ADC. This type of transfer function is also known as "with no true zero".
In an ideal ADC the transition voltages are equally spaced by $Q$, the ideal code bin width, given by

$$
Q=\frac{2 \cdot F S}{2^{n_{b}}}
$$

and the ideal values of the transition voltages are

$$
T_{k}^{\text {ideal }}=-F S+k \cdot Q \text { with } k=1 \ldots 2^{n_{b}}-1,
$$

where FS is the ADC Full Scale voltage.

In a real ADC, however, the transition voltages are different from the ideal ones due to manufacturing defects and environmental conditions. It is thus important, for the user of an ADC, to know how close a given ADC is behaving in relation to its ideal, and expected, behavior. To that effect several types ADC tests can be used. The most common one, for the estimation of an ADC transfer function, is the Histogram Test (also known as Code Density Test) [1-9]. This is a statistical test where a sinusoidal stimulus signal is applied and a large amount of samples are required. From the digital output codes obtained, the behavior of the ADC is inferred and its transfer function estimated, that is, the values of the transition voltages are estimated.

Fig. 1 represents the transfer function of a symmetrical bipolar ADC, also known as a bipolar with no true zero transfer function because there is no step whose middle point is zero. It is used mainly in theoretical considerations where the symmetry of transition voltages eases the derivations. There are, however other types of transfer functions like the bipolar with true zero and the unipolar, which have different values of transition voltages. The derivations carried on in this paper can easily be adapted to those cases if needed.

To better evaluate the performance of an ADC, different parameters are used to express the transfer function, namely, the gain, offset error, integral and differential non linearity. The first two, gain and offset error, express the transfer function as a straight line that ideally goes through the middle point of the quantification steps (black circles in Fig. 1). The latter two, integral and differential non linearity, pertain to the difference between the ideal and real transition voltages and code bin widths respectively.

In this paper we study the influence that the 
presence of additive noise and acquisition of a finite number of samples has on the precision of the estimative of ADC gain and offset error determined with the Histogram Method. The goal is to derive expressions that let us know what is the precision of those estimates. In the case of ADC testing, as in any measurement system, the result of the measurement should be accompanied by confidence intervals that give an idea about the uncertainty of the measurement result. In this paper we will concentrate our efforts in determining the standard deviation of the estimated gain and offset error which will allow us to specify the correspondent confidence intervals.

In section II we will present the definitions of gain and offset error. In the following section (III) we address the precision of the parameters of estimated from linear regression which is used to estimate the ADC gain and offset error. In section IV we derive the covariance of the transition voltages estimated with the histogram method with the purpose of determining the standard deviation of the ADC gain and offset error which is done in section V. In section VI we present experimental results that validate the analytical derivations and approximations done. Finally, in section VIII, we present the conclusions reached with this work.

\section{INDEPENDENTLY BASED GAIN AND OFFSET ERROR}

There are two ways that are traditionally used to define an ADC gain and offset error. They are the Terminal Based Definition and the Independently Based Definition [10]. In this paper we will focus our attention on the second one, leaving the other one for a different publication.

In the Independently Based Definition, the gain and offset error are defined as the two scalars that when used to multiply the estimated transition voltages, $\hat{T}_{k}$, (gain, $G$ ) and add to the result of the multiplication (offset error, $O$ ) lead to corrected transition voltages

$$
\hat{T}_{k}^{\text {corr }}=G \cdot \widehat{T}_{k}+O
$$

that are as close as possible to the ideal transition voltages in a least square sense.

The way traditionally used to determine the independently based ADC gain and offset error is to use a linear regression procedure to fit the estimated transition voltages to the ideal ones:

$$
T_{k}^{\text {ideal }}=\hat{G} \cdot \hat{T}_{k}+\widehat{O} \text {. }
$$

The estimated gain is the slope of the fitted straight line and the estimated offset error is the point of intersection of that straight line with the vertical axis (axis of the ideal transition voltages).

In the following section we will present the expressions for the standard deviation of the estimated gain and offset error obtained from the linear regression.

\section{Precision of the Parameters of Linear REGRESSION}

Consider an independent variable $x$ (not random) and a dependent variable $y$ that are related in the following way:

$$
y_{i}=\beta_{0}+\beta_{1} \cdot x_{i}+u_{i} \quad, \quad i=1,2, \ldots, I,
$$

where $\beta_{1}$ is the slope parameter and $\beta_{0}$ is the intercept parameter. The term $u_{i}$ represents an error, that is, it represents other factors, other than $x$, that influence $y$.

From a set of values of $x$ and $y$ it is possible to use the following least squares estimators for the two parameters [11]:

$$
\begin{aligned}
& \hat{\beta}_{1}=\frac{\sum_{i=1}^{I}\left(x_{i}-\bar{x}\right)\left(y_{i}-\bar{y}\right)}{\sum_{i=1}^{I}\left(x_{i}-\bar{x}\right)^{2}}=\frac{s_{x y}}{s_{x x}} . \\
& \hat{\beta}_{0}=\bar{y}-\widehat{\beta}_{1} \bar{x}
\end{aligned}
$$

The distance between the points $y_{i}$ and the fitted straight line is called residual $\left(\hat{u}_{i}\right)$ :

$$
\hat{u}_{i}=y_{i}-\widehat{\beta}_{0}-\widehat{\beta}_{1} \cdot x_{i} .
$$

The least square estimators (6) are the best linear unbiased estimators, according to the Gauss-Markov Theorem [12] if the following assumptions are satisfied:

$$
\begin{aligned}
& E\left[u_{i}\right]=0 \\
& \operatorname{var}\left[u_{i}\right]=\sigma^{2}<\infty . \\
& \operatorname{cov}\left[u_{i}, u_{j}\right]=0
\end{aligned}
$$

These assumptions mean that the errors must have zero mean, be uncorrelated and must all have the same variance (homoskedasticity).

Unfortunately, in the case of the determination of the independently based ADC gain and offset error with the Histogram Test, in the presence of additive noise, none of these assumptions is valid. The estimated transition voltages do not have zero mean [13], do not all have the same variance [8] and are correlated as will be shown in the following section.

As a consequence, the least squares estimators (6) are not the best ones. Since the variances of the estimated voltages can be computed, it is possible to define other estimators that are better than these by using weighted least square estimation. However, since traditionally the normal least square estimators are used, we consider it important to study them even though they are not the best estimators possible. We plan, in the future, to extend the study presented here to other estimators based in weighted least square procedure.

So, given the estimators (6), we want to determine the standard deviation of the parameters $\beta$. The parameter $\beta_{1}$ in (6) can be written as

$$
\widehat{\beta}_{1}=\frac{1}{s_{x x}} \sum_{i=1}^{I}\left(x_{i}-\bar{x}\right)\left(y_{i}-\bar{y}\right)=\frac{1}{s_{x x}} \sum_{i=1}^{I}\left(x_{i}-\bar{x}\right) y_{i} .
$$


The variance of the estimator of $\beta_{1}$ is, using the properties of variance [14],

$$
\begin{aligned}
& \operatorname{var}\left[\hat{\beta}_{1}\right]=\frac{1}{s_{x x}^{2}} \operatorname{var}\left[\sum_{i=1}^{I}\left(x_{i}-\bar{x}\right) y_{i}\right]= \\
& =\frac{1}{s_{x x}^{2}} \sum_{k=1}^{I} \sum_{l=1}^{I} \operatorname{cov}\left[\left(x_{k}-\bar{x}\right) y_{k},\left(x_{l}-\bar{x}\right) y_{l}\right]=. \\
& =\frac{1}{s_{x x}^{2}} \sum_{k=1}^{N} \sum_{l=1}^{N}\left(x_{k}-\bar{x}\right)\left(x_{l}-\bar{x}\right) \operatorname{cov}\left[y_{k}, y_{l}\right]
\end{aligned}
$$

For the estimated offset error, using (6) and considering $\bar{x}=0$, leads to

$\operatorname{var}\left[\hat{\beta}_{0}\right]=\frac{1}{I^{2}} \operatorname{var}\left[\sum_{i=1}^{I} y_{i}\right]=\frac{1}{I^{2}} \sum_{k=1}^{I} \sum_{l=1}^{I} \operatorname{cov}\left[y_{k}, y_{l}\right]$.

Since the independent variable, $x$, is considered as non random and given what was said in the previous section about the definition of independently based gain and offset error we will change equation (4) to

$$
\widehat{T}_{k}=\widehat{G}^{\prime} \cdot T_{k}^{\text {ideal }}+\widehat{O}^{\prime}
$$

where the parameters $G^{\prime}$ and $O^{\prime}$ are related to the ADC gain and offset error by

$$
\widehat{G}=\frac{1}{\widehat{G}^{\prime}} \text { and } \widehat{O}=-\frac{\widehat{O}^{\prime}}{\widehat{G}^{\prime}} .
$$

From [15] we can write

$$
\operatorname{var}[\widehat{G}] \approx \operatorname{var}\left[\widehat{G}^{\prime}\right] \text { and } \operatorname{var}[\widehat{O}] \approx \operatorname{var}\left[\widehat{O}^{\prime}\right]
$$

having approximated the mean of $\widehat{G}^{\prime}$ by its ideal value of 1 .

Comparing (5) with (12) we have $y=\hat{T}$ and $x=T^{\text {ideal }}$. To compute (10) and (11) it is necessary to derive the covariance of the estimated transition voltages which is done in the following section.

\section{COVARIANCE OF THE ESTIMATED TRANSITION VOLTAGES}

\section{A. Histogram Test}

In the Histogram Test usually a sinusoidal stimulus signal is applied to the ADC input:

$$
s(t)=C-A \cdot \cos (2 \pi \cdot f \cdot t+\varphi) .
$$

Due to the presence of additive noise in the stimulus signal and generated in the ADC itself we can consider the sampled voltage to be

$$
v_{j}=s\left(\frac{j}{f_{s}}\right)+n_{V} \quad, j=0,1, \ldots, M-1 .
$$

where $n_{V}$ is a random variable that represents additive white Gaussian noise (AWGN) and $M$ is the number of samples acquired. Inserting (15) into (16) leads to

$$
v_{j}=C-A \cdot \cos \left(2 \pi \cdot f \cdot \frac{j}{f_{s}}+\varphi\right)+n_{V} .
$$

In order to simplify the derivations we will introduce the following normalized variables:

$$
u_{j}=\frac{v_{j}-C}{A}, n=\frac{n_{V}}{A} \quad, \quad \gamma_{j}=2 \pi \frac{f}{f_{s}} t_{j}+\varphi .
$$

Using these variables we can write the normalized sampled voltage, from (17), as

$$
u_{j}=-\cos \left(\gamma_{j}\right)+n \text {. }
$$

The $M$ samples acquired are used to construct the cumulative histogram, $c_{k}$, which is the number of samples with output code equal to or lower than $k$. The transition voltages are then estimated with [1]

$$
\hat{T}_{k}=C-A \cdot \cos \left(\frac{\pi}{M} C_{k}\right)
$$

or, using a normalized transition voltage,

$$
\widehat{U}_{k}=\frac{\widehat{T}_{k}-C}{A}=-\cos \left(\frac{\pi}{M} c_{k}\right) \text {. }
$$

\section{B. Probability of a sample having a given code}

To compute the covariance of the transition voltages we will need first to compute the covariance of the number of counts of the cumulative histogram, $c_{k}$. To that effect we will introduce a binomial variable, $w_{k}$, which assumes the value 1 if a sample belongs to class $k$ of the cumulative histogram and 0 otherwise. The probability that a given sample, with phase $\gamma_{j}$, belongs to $c_{k}$ is the probability that the sampled voltage (with phase $\gamma_{j}$ ) is lower than the transition voltage $U_{k}[8]$

$$
p_{k}\left(\gamma_{j}\right)=\frac{1}{2}+\frac{1}{2} \operatorname{erf}\left(\frac{U_{k}+\cos \left(\gamma_{j}\right)}{\sqrt{2} \cdot \sigma_{n}}\right) .
$$

The definition of covariance is [15]

$$
\operatorname{cov}\left[w_{k}, w_{l}\right]=E\left[w_{k} w_{l}\right]-E\left[w_{k}\right] E\left[w_{l}\right] .
$$

The expected value, $E\left[w_{k} w_{l}\right]$, of the product of $w_{k}$ and $w_{l}$ is the probability that a sample belongs simultaneously to $c_{k}$ and $c_{l}$. which is to say that it is the probability that the sampled voltage is lower than both $U_{k}$ and $U_{l}$. This probability is just the probability that the sampled voltage is lower than the minimum of $U_{k}$ and $U_{l}$ :

$$
E\left[w_{k} w_{l} \mid \gamma_{j}\right]=\left\{\begin{array}{ll}
p_{k}\left(\gamma_{j}\right), & U_{k}<U_{l} \\
p_{l}\left(\gamma_{j}\right), & \text { otherwise }
\end{array} .\right.
$$

Since $w$ is a binomial variable its expected value is equal to the probability of $w=1$ which is

$$
E\left[w_{k} \mid \gamma_{j}\right]=p_{k}\left(\gamma_{j}\right) \text { and } E\left[w_{l} \mid \gamma_{j}\right]=p_{l}\left(\gamma_{j}\right)
$$

Inserting (24) and (25) into (23) leads to $\operatorname{cov}\left[w_{k} w_{l} \mid \gamma_{j}\right]= \begin{cases}p_{k}\left(\gamma_{j}\right)\left[1-p_{l}\left(\gamma_{j}\right)\right], & U_{k}<U_{l} \\ p_{l}\left(\gamma_{j}\right)\left[1-p_{k}\left(\gamma_{j}\right)\right], & \text { otherwise }\end{cases}$

\section{Conditional Covariance of the Cumulative Histogram}

The number of counts of the cumulative histogram, 
$c_{k}$, is just the sum of variable $w_{k}$ for all samples acquired:

$$
c_{k}=\sum_{j=0}^{M-1} w_{k, j} .
$$

The covariance between the summations of two variables is the double summation of the covariance of those variables [14]:

$$
\begin{aligned}
& \operatorname{cov}\left[c_{k}, c_{l} \mid \varphi\right]=\operatorname{cov}\left[\sum_{j=0}^{M-1} w_{k \mid \gamma_{j}}, \sum_{i=0}^{M-1} w_{l \mid \gamma_{i}}\right]= \\
& =\sum_{j=0}^{M-1} \sum_{i=0}^{M-1} \operatorname{cov}\left[w_{k \mid \gamma_{j}}, w_{l \mid \gamma_{i}}\right]
\end{aligned}
$$

A sample having or not a certain output code is independent of another sample, with a different phase, of having the same or another output code. From this it follows that the covariance in (28) is only different from 0 when $i=j$. Eq. (28) can thus be written as

$$
\operatorname{cov}\left[c_{k}, c_{l} \mid \varphi\right]=\sum_{j=0}^{M-1} \operatorname{cov}\left[w_{k \mid \gamma_{j}}, w_{l \mid \gamma_{j}}\right] \text {. }
$$

\section{Total Covariance of the Cumulative Histogram}

Usually the stimulus signal and the sampling signal are not synchronized which means that the phase at the origin of the stimulus signal, $\varphi$, can assume any value. Considering that it has a uniform distribution in the interval $[-\pi, \pi]$ the total covariance of the number of counts of the cumulative histogram can be computed from the conditional covariance given by (29) [14]:

$$
\begin{aligned}
& \operatorname{cov}\left[c_{k}, c_{l}\right]= \\
& =E\left\{\operatorname{cov}\left[c_{k}, c_{l} \mid \varphi\right]\right\}+\operatorname{cov}\left[E\left[c_{k} \mid \varphi\right], E\left[c_{l} \mid \varphi\right]\right]
\end{aligned}
$$

The first term of the right side of eq. (30) is

$$
E\left\{\operatorname{cov}\left[c_{k}, c_{l} \mid \varphi\right]\right\}=\frac{1}{2 \pi} \int_{-\pi}^{\pi} \operatorname{cov}\left[c_{k}, c_{l} \mid \varphi\right] d \varphi .
$$

Inserting (29) leads to

$$
E\left\{\operatorname{cov}\left[c_{k}, c_{l} \mid \varphi\right]\right\}=\frac{1}{2 \pi} \int_{-\pi}^{\pi} \sum_{j=0}^{M-1} \operatorname{cov}\left[w_{k \mid \gamma_{j}}, w_{l \mid \gamma_{j}}\right] d \varphi
$$

Considering the definition of $\gamma_{j}$, given by (18), and that (22) is periodic on $\gamma_{j}$, eq. (32) becomes

$$
E\left\{\operatorname{cov}\left[c_{k}, c_{l} \mid \varphi\right]\right\}=\frac{M}{2 \pi} \int_{-\pi}^{\pi} \operatorname{cov}\left[w_{k \mid \gamma_{j}}, w_{l \mid \gamma_{j}}\right] d \varphi .
$$

Inserting (26) leads to

$$
\begin{aligned}
& E\left\{\operatorname{cov}\left[c_{k}, c_{l} \mid \varphi\right]\right\}= \\
& =\frac{M}{2 \pi}\left\{\begin{array}{l}
\int_{-\pi}^{\pi} p_{k}\left(\gamma_{j}\right)\left[1-p_{l}\left(\gamma_{j}\right)\right] d \varphi, \quad U_{k}<U_{l} . \\
\int_{-\pi}^{\pi} p_{l}\left(\gamma_{j}\right)\left[1-p_{k}\left(\gamma_{j}\right)\right] d \varphi, \text { otherwise }
\end{array}\right.
\end{aligned}
$$

In relation to the second term of the right side of (30), we can use the definition of covariance to write

$$
\begin{aligned}
& \operatorname{cov}\left[E\left[c_{k} \mid \varphi\right], E\left[c_{l} \mid \varphi\right]\right]= \\
& E\left\{E\left[c_{k} \mid \varphi\right] \cdot E\left[c_{l} \mid \varphi\right]\right\}-E\left\{E\left[c_{k} \mid \varphi\right]\right\} \cdot E\left\{E\left[c_{l} \mid \varphi\right]\right\}
\end{aligned} .
$$

Considering the uniform distribution of $\varphi$ between $-\pi$ and $\pi$ leads to

$$
\begin{aligned}
& \operatorname{cov}\left[E\left[c_{k} \mid \varphi\right], E\left[c_{l} \mid \varphi\right]\right]= \\
& \quad \frac{1}{2 \pi} \int_{-\pi}^{\pi} E\left[c_{k} \mid \varphi\right] \cdot E\left[c_{l} \mid \varphi\right] d \varphi- \\
& \frac{1}{(2 \pi)^{2}} \int_{-\pi}^{\pi} E\left[c_{k} \mid \varphi\right] d \varphi \cdot \int_{-\pi}^{\pi} E\left[c_{l} \mid \varphi\right] d \varphi
\end{aligned} .
$$

The conditional expected value of $c_{k}$ is the sum of the conditional expected values of all variables $w_{k}$

$$
E\left[c_{k} \mid \varphi\right]=\sum_{j=0}^{M-1} E\left[w_{k, j}\right]=\sum_{j=0}^{M-1} p_{k}\left(\gamma_{j}\right) .
$$

Substituting in (36) leads to

$$
\begin{aligned}
& \operatorname{cov}\left[E\left[c_{k} \mid \varphi\right], E\left[c_{l} \mid \varphi\right]\right]= \\
& \frac{1}{2 \pi} \int_{-\pi}^{\pi} \sum_{j=0}^{M-1} p_{k}\left(\gamma_{j}\right) \cdot \sum_{j=0}^{M-1} p_{l}\left(\gamma_{j}\right) d \varphi- \\
& -\frac{1}{(2 \pi)^{2}} \int_{-\pi}^{\pi} \sum_{j=0}^{M-1} p_{k}\left(\gamma_{j}\right) d \varphi \cdot \int_{-\pi}^{\pi} \sum_{j=0}^{M-1} p_{l}\left(\gamma_{j}\right) d \varphi
\end{aligned} .
$$

Using the same arguments employed when deriving eq. (33) we can write

$$
\begin{aligned}
& \operatorname{cov}\left[E\left[c_{k} \mid \varphi\right], E\left[c_{l} \mid \varphi\right]\right]= \\
& \frac{1}{2 \pi} \int_{-\pi}^{\pi} \sum_{j=0}^{M-1} p_{k}\left(\gamma_{j}\right) \cdot \sum_{j=0}^{M-1} p_{l}\left(\gamma_{j}\right) d \varphi-. \\
& -\frac{M^{2}}{(2 \pi)^{2}} \int_{-\pi}^{\pi} p_{k}(\varphi) \cdot \int_{-\pi}^{\pi} p_{l}(\varphi) d \varphi
\end{aligned}
$$

Inserting (34) and (39) into (30) leads to the expression for the covariance of the number of counts of the cumulative histogram:

$$
\begin{aligned}
& \operatorname{cov}\left[c_{k}, c_{l}\right]= \\
& \frac{M}{2 \pi}\left\{\begin{array}{l}
\int_{-\pi}^{\pi} p_{k}\left(\gamma_{j}\right)\left[1-p_{l}\left(\gamma_{j}\right)\right] d \varphi, U_{k}<U_{l} \\
\int_{-\pi}^{\pi} p_{l}\left(\gamma_{j}\right)\left[1-p_{k}\left(\gamma_{j}\right)\right] d \varphi, \text { otherwise }
\end{array}+\right. \\
& +\frac{1}{2 \pi} \int_{-\pi}^{\pi} \sum_{j=0}^{M-1} p_{k}\left(\gamma_{j}\right) \cdot \sum_{j=0}^{M-1} p_{l}\left(\gamma_{j}\right) d \varphi- \\
& -\frac{M^{2}}{(2 \pi)^{2}} \int_{-\pi}^{\pi} p_{k}(\varphi) \cdot \int_{-\pi}^{\pi} p_{l}(\varphi) d \varphi
\end{aligned}
$$

As can be seen in Fig. 2, the covariance of the expected values of the number of counts of the cumulative histogram tends to 0 as the standard deviation of noise increases. This can also be seen for the variance of the expected values of the number of counts as shown in [8]. The condition found in [8] was that this term can be considered approximately null for situations where $M \cdot \sigma_{n}>1$. To give an idea of the actual values one might encounter for this product, consider a stimulus signal with an amplitude equal to the ADC Full Scale $(A=F S)$ and that the additive noise standard deviation is $0.5 \operatorname{LSB}\left(\sigma_{n_{V}}=Q / 2\right)$. The referred condition is thus 


$$
M \cdot \sigma_{n}>1 \Leftrightarrow M \cdot \frac{Q}{2 A}>1 \Leftrightarrow M>2^{n b}
$$

which corresponds to having more than 1 sample per output code. Even if the additive noise is greater, for instance, 2 LSB, this condition corresponds to at least 4 samples per output code which is a common situation in actual tests using the Histogram Method. This approximation will be used later to derive an approximate expression for the variance of the estimated gain and offset error.

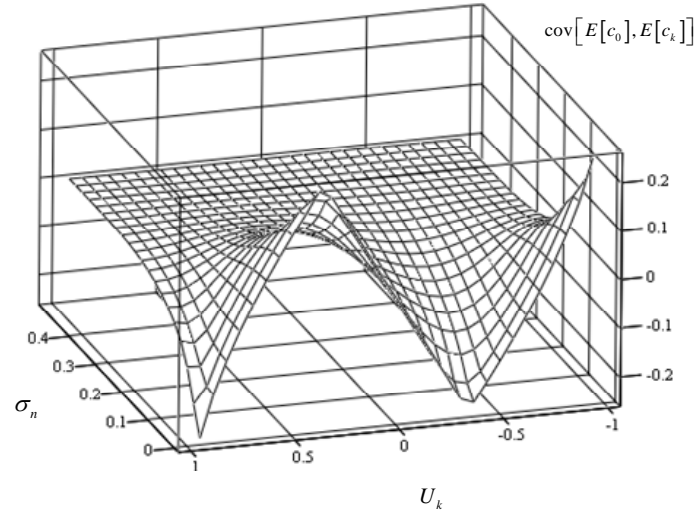

Fig. 2 - Representation of the covariance between the expected value of the number of counts of class 0 and of class $k$ of the cumulative histogram as a function of the normalized transition voltage of class $k$ and the standard deviation of additive noise. $M=4$.

\section{E. Covariance of the Transition Voltages}

The estimated value of the transition voltages is obtained from the number of counts of the cumulative histogram with (20). The covariance of the estimated transition voltages is given by [15]

$$
\left.\left.\operatorname{cov}\left[\hat{T}_{k}, \hat{T}_{l}\right] \approx \frac{d \hat{T}_{k}}{d c_{k}}\right|_{\hat{T}_{k}=\mu_{\hat{T}_{k}}} \frac{d \hat{T}_{l}}{d c_{l}}\right|_{\hat{T}_{l}=\mu_{\hat{T}_{l}}} \operatorname{cov}\left[c_{k}, c_{l}\right] .
$$

Differentiating (20) and inserting into (42) leads to

$$
\begin{aligned}
& \operatorname{cov}\left[\hat{T}_{k}, \hat{T}_{l}\right] \approx\left(\frac{\pi}{M}\right)^{2} \sqrt{A^{2}-\mu_{\hat{T}_{k}}^{2}} \times \\
& \times \sqrt{A^{2}-\mu_{\hat{T}_{l}}^{2}} \operatorname{cov}\left[c_{k}, c_{l}\right]
\end{aligned}
$$

We considered, to simplify the computations, that the stimulus signal has no offset $(C=0)$. The same results would have been obtained if the stimulus signal had an offset.

\section{GAIN PRECISION}

The variance of the estimated ADC gain is given by (10), with $y=T$, as seen previously. Inserting (43) gives rise to

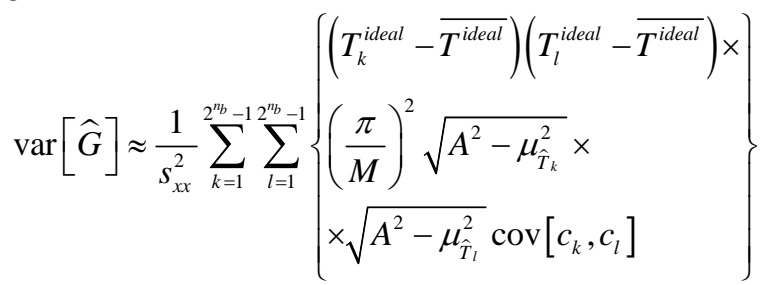

Making the variable substitution $T=U A$, leads to

$$
\begin{aligned}
& \operatorname{var}[\hat{G}] \approx \frac{A^{4}}{S_{x x}^{2}}\left(\frac{\pi}{M}\right)^{2} \times \\
& \times \sum_{k=1}^{2^{n_{b}}-1} \sum_{l=1}^{2^{n_{b}}-1}\left\{\begin{array}{l}
\left(U_{k}^{\text {ideal }}-\overline{U^{\text {ideal }}}\right)\left(U_{l}^{\text {ideal }}-\overline{U^{\text {ideal }}}\right) \times \\
\sqrt{1-\mu_{\hat{U}_{k}}^{2}} \sqrt{1-\mu_{\hat{U}_{l}}^{2}} \operatorname{cov}\left[c_{k}, c_{l}\right]
\end{array}\right\}
\end{aligned}
$$

Using (6) and considering that $x$ represents the ideal transition voltage, we can write

$$
S_{x x}=\sum_{i=1}^{2^{n b}-1}\left(T_{i}^{\text {ideal }}-\overline{T^{\text {ideal }}}\right)^{2} .
$$

For a sufficient number of bits (in general more than 4) we can approximate the summation in (46) by an integral:

$$
S_{x x} \approx \frac{2^{n_{b}}-1}{2(F S-Q)} \int_{-F S+Q}^{F S-Q} T^{2} d T=\frac{2^{n_{b}}-1}{3}(F S-Q)^{2} .
$$

We considered that it was a bipolar with no zero ADC, which means that the transition voltages are symmetric and thus their mean is null. Inserting into (45) leads to

$$
\begin{aligned}
& \operatorname{var}[\widehat{G}] \approx\left(\frac{\pi}{M}\right)^{2} \frac{9 A^{4}}{\left(2^{n_{b}}-1\right)^{2}(F S-Q)^{4}} \times \\
& \times \sum_{k=1}^{2^{n_{b}}-1} \sum_{l=1}^{2^{n_{b}}-1}\left\{\begin{array}{l}
\left(U_{k}^{\text {ideal }}-\overline{U^{\text {ideal }}}\right)\left(U_{l}^{\text {ideal }}-\overline{U^{\text {ideal }}}\right) \times \\
\sqrt{1-\mu_{\widehat{U}_{k}}^{2}} \sqrt{1-\mu_{\widehat{U}_{I}}^{2}} \operatorname{cov}\left[c_{k}, c_{l}\right]
\end{array}\right\}
\end{aligned}
$$

The double summation in (48) can also be approximated by a double integration in $U_{k}$ and $U_{l}$ :

$$
\begin{aligned}
& \operatorname{var}[\hat{G}] \approx \frac{9}{4}\left(\frac{\pi}{M}\right)^{2} \times\left(\frac{A}{F S-Q}\right)^{4} \\
& \times \int_{-1}^{1} \int_{-1}^{1}\left\{\begin{array}{l}
U_{k} U_{l} \sqrt{1-\mu_{\hat{U}_{k}}^{2}} \times \\
\times \sqrt{1-\mu_{\hat{U}_{l}}^{2}} \operatorname{cov}\left[C_{k}, C_{l}\right]
\end{array}\right\} d U_{k} d U_{l}
\end{aligned}
$$

Note that the integration limits should be $(-F S+Q) / A$ and $(F S-Q) / A$ which are the normalized values of the first and last transition voltage. In practice the value of stimulus amplitude used, $A$, is slightly larger than FS-Q because the ADC must be overdriven to minimize the bias introduced in the transition voltage estimates due to the presence of additive noise [13]. We chose to use the limits \pm 1 in the integrals in (49) in order to have its value independent of both $A$ and FS. As a consequence the value of the integration becomes slightly smaller.

Using the approximation justified in the previous section, $M \cdot \sigma_{n}>1$, we can substitute the covariance in (49) by the expected value of the covariance term given by (34) 


$$
\begin{aligned}
& \operatorname{var}[\hat{G}] \approx \frac{9}{4} \frac{\pi}{M}\left(\frac{A}{F S-Q}\right)^{4} \times \\
& \times \int_{-1}^{1} \int_{-1}^{U_{l}}\left\{\begin{array}{l}
U_{k} U_{l} \sqrt{1-U_{k}^{2}} \sqrt{1-U_{l}^{2}} \times \\
\int_{-\pi}^{\pi} p_{k}(\varphi)\left[1-p_{l}(\varphi)\right] d \varphi
\end{array}\right\} d U_{k} d U_{l}
\end{aligned}
$$

In the previous expression we have taken into account that for small amounts of additive noise $\left(\sigma_{n}<0.1\right)$ usually encountered in practical situations, and considering the use of overdrive, we can assume the mean of the estimated transition voltages to be unbiased [13].

Inserting (22) into (50) leads to

$$
\begin{aligned}
& \operatorname{var}[\hat{G}] \approx \frac{9}{4 M} \times\left(\frac{A}{F S-Q}\right)^{4} \\
& \times \int_{-1-1}^{1} \int_{U_{l}}\left\{\begin{array}{l}
\pi U_{k} U_{l} \sqrt{1-U_{k}^{2}} \sqrt{1-U_{l}^{2}} \times \\
\times \int_{-\pi}^{\pi}\left[\frac{1}{2}+\frac{1}{2} \operatorname{erf}\left(\frac{U_{k}+\cos (\varphi)}{\sqrt{2} \cdot \sigma_{n}}\right)\right] \times \\
\times\left[\frac{1}{2}-\frac{1}{2} \operatorname{erf}\left(\frac{U_{l}+\cos (\varphi)}{\sqrt{2} \cdot \sigma_{n}}\right)\right] d \varphi
\end{array}\right\} d U_{k} d U_{l}
\end{aligned}
$$

These integrals were solved numerically and an approximated value of $1.21 \sigma_{n}^{2}$ was obtained.

$$
\operatorname{var}[\hat{G}] \approx \frac{9}{4} \frac{1.21 \sigma_{n}^{2}}{M}\left(\frac{A}{F S-Q}\right)^{4} .
$$

Using (18) we can write, for the estimated gain standard deviation,

$$
\sigma_{\hat{G}} \approx 1.1 \frac{3}{2} \frac{A}{(F S-Q)^{2}} \frac{\sigma_{n_{v}}}{\sqrt{M}} .
$$

\section{OfFSET ERROR PrECISION}

The variance of the estimated ADC offset is given by (11) with $y=T$, as seen previously. Inserting (43) gives rise to

$$
\operatorname{var}[\hat{O}] \approx \frac{\sum_{k=1}^{2^{n_{b}}-12^{2_{b}-1}}\left\{\begin{array}{l}
\left(\frac{\pi}{M}\right)^{2} \sqrt{A^{2}-\mu_{\hat{T}_{k}}^{2}} \times \\
\sqrt{A^{2}-\mu_{\hat{T}_{l}}^{2}} \operatorname{cov}\left[c_{k}, c_{l}\right]
\end{array}\right\}}{\left(2^{n_{b}}-1\right)^{2}} .
$$

Making the variable substitution $T=U A$, leads to

$$
\begin{aligned}
& \operatorname{var}[\hat{O}] \approx \frac{A^{2}}{\left(2^{n_{b}}-1\right)^{2}}\left(\frac{\pi}{M}\right)^{2} \times \\
& \times \sum_{k=1}^{2^{n_{b}}-12^{n_{b}}-1} \sqrt{1-\mu_{\hat{U}_{k}}^{2}} \sqrt{1-\mu_{\hat{U}_{l}}^{2}} \operatorname{cov}\left[c_{k}, c_{l}\right]
\end{aligned}
$$

The double summation in (55) can be approximated, for an ADC with a sufficiently high number of bits, by a double integration between the first and last normalized transition voltages:

$$
\begin{aligned}
& \operatorname{var}[\hat{O}] \approx \frac{A^{2}}{4(F S-Q)^{2}}\left(\frac{\pi}{M}\right)^{2} \times A^{2} \\
& \times \int_{-\frac{F S-Q}{A}-\frac{F S-Q}{A}}^{\frac{F S-Q}{A}}\left\{\sqrt{1-\mu_{\hat{U}_{k}}^{2}} \sqrt{1-\mu_{\hat{U}_{l}}^{2}} \operatorname{cov}\left[c_{k}, c_{l}\right]\right\} d U_{k} d U_{l}
\end{aligned}
$$

As was done for the estimated gain variance, we will replace the limits of integration with \pm 1 :

$$
\begin{aligned}
& \operatorname{var}[\hat{O}] \approx \frac{A^{2}}{4(F S-Q)^{2}}\left(\frac{\pi}{M}\right)^{2} \times A^{2} \\
& \times \int_{-1}^{1} \int_{-1}^{1}\left\{\sqrt{1-\mu_{\hat{U}_{k}}^{2}} \sqrt{1-\mu_{\hat{U}_{l}}^{2}} \operatorname{cov}\left[c_{k}, c_{l}\right]\right\} d U_{k} d U_{l}
\end{aligned}
$$

Using the approximation justified previously, $M \cdot \sigma_{n}>1$, we can substitute the covariance in (56) by the expected value of the covariance term given by (34),

$$
\begin{aligned}
& \operatorname{var}[\hat{O}] \approx \frac{A^{4}}{4(F S-Q)^{2}} \frac{\pi}{M} \times \\
& \times \int_{-1}^{1} \int_{-1}^{U_{l}}\left\{\begin{array}{l}
\sqrt{1-U_{k}^{2}} \sqrt{1-U_{l}^{2}} \times \\
\times \int_{-\pi}^{\pi} p_{k}(\varphi)\left[1-p_{l}(\varphi)\right] d \varphi
\end{array}\right\} d U_{k} d U_{l}
\end{aligned}
$$

Note that the limits of integration on $U_{k}$ have changed due to the piecewise definition used in (34).

In the previous expression we have taken into account that for small amounts of additive noise $\left(\sigma_{n}<0.1\right)$ usually encountered in practical situations, and considering the use of overdrive, we can assume the mean of the estimated transition voltages to be unbiased [13].

Inserting (22) leads to

$$
\begin{aligned}
& \operatorname{var}[\hat{O}] \approx \frac{A^{4}}{4(F S-Q)^{2}} \frac{1}{M} \times \\
& \times \int_{-1-1}^{1} \int_{-1}^{U_{l}}\left\{\begin{array}{c}
\pi \sqrt{1-U_{k}^{2}} \sqrt{1-U_{l}^{2}} \times \\
\times \int_{-\pi}^{\pi}\left[\frac{1}{2}+\frac{1}{2} \operatorname{erf}\left(\frac{U_{k}+\cos (\varphi)}{\sqrt{2} \cdot \sigma_{n}}\right)\right] \times \\
\times\left[\frac{1}{2}-\frac{1}{2} \operatorname{erf}\left(\frac{U_{l}+\cos (\varphi)}{\sqrt{2} \cdot \sigma_{n}}\right)\right] d \varphi
\end{array}\right\} d U_{k} d U_{l}
\end{aligned}
$$

These integrals were solved numerically and a value of approximately $5 \sigma_{n}^{2}$ was obtained.

$$
\operatorname{var}[\hat{O}] \approx \frac{A^{4}}{4(F S-Q)^{2}} \frac{5 \sigma_{n}^{2}}{M} .
$$

Using (47) and (18) we can write, for the estimated gain standard deviation,

$$
\sigma_{\hat{o}} \approx 1.1 \frac{A}{F S-Q} \frac{\sigma_{n_{v}}}{\sqrt{M}} .
$$




\section{EXPERIMENTAL RESULTS}

To demonstrate the validity of the expressions presented here we tested a 12-bit ADC using the Standard Histogram Test. Only the 8 most significant bits were used to so that the ADC could be considered ideal. Using a Monte Carlo procedure with 1000 repetitions $(N)$ we computed the standard deviation of the estimated independently based gain and offset error. We varied the additive noise standard deviation, number of samples, stimulus signal amplitude and ADC Full Scale range.

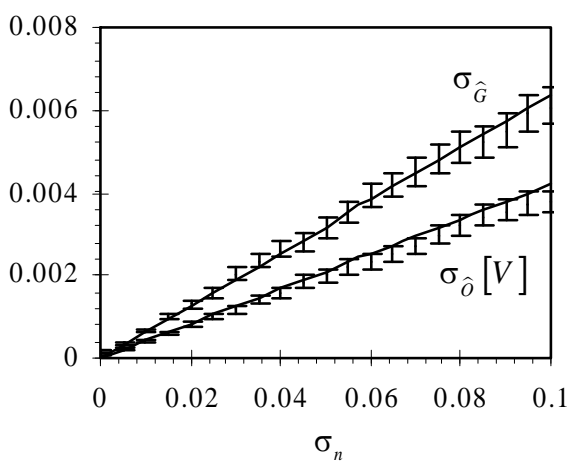

Fig. 3 - Representation of the estimated gain and offset error standard deviations as a function of the normalized additive noise standard deviation. We considered $n_{b}=8, A=1.2 \mathrm{~V}, F S=1 \mathrm{~V}, M=1000$, $f=200 \mathrm{~Hz}, f_{\mathrm{s}}=200 \mathrm{kHz}$ and $N=1000$. The solid lines represent the approximation given by (53) and (61), and the vertical bars represent the experimental results.

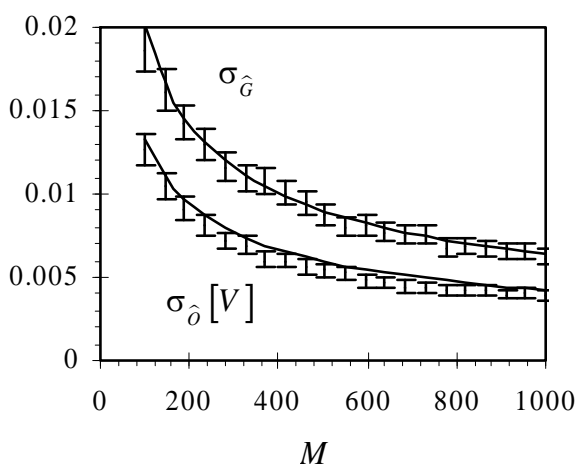

Fig. 4 - Representation of the estimated gain and offset error standard deviations as a function of the number of samples. We considered $n_{b}=8, A=1.2 \mathrm{~V}, F S=1 \mathrm{~V}, \sigma_{n}=0.1, f=f_{s} / M, f_{s}=200 \mathrm{kHz}$ and $N=1000$. The solid lines represent the approximation given by (53) and (61), and the vertical bars represent the experimental results.

The results obtained for the standard deviation of the estimated ADC gain and offset error are presented in Fig. 3 through Fig. 6.

The vertical bars in the figures translate the $99.9 \%$ confidence interval to account for the Monte Carlo uncertainty due to the 1000 test repetitions $(N)$ carried out.

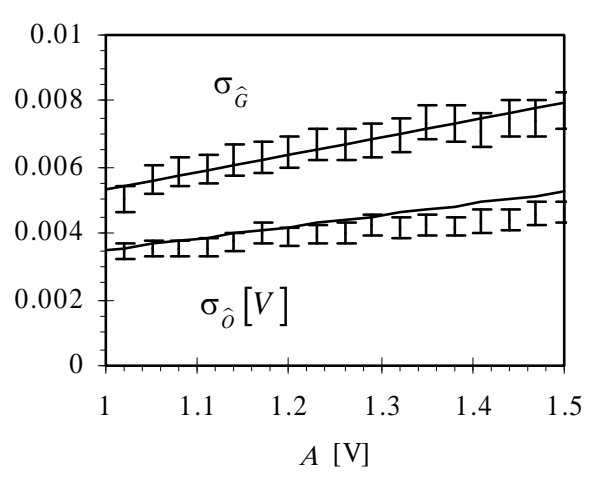

Fig. 5 - Representation of the estimated gain and offset error standard deviations as a function of the stimulus signal amplitude. We considered $n_{b}=8, M=1000, F S=1 \mathrm{~V}, \sigma_{n}=0.1, f=200 \mathrm{~Hz}, f_{s}=200 \mathrm{kHz}$ and $N=1000$. The solid lines represent the approximation given by

(53) and (61), and the vertical bars represent the experimental results.

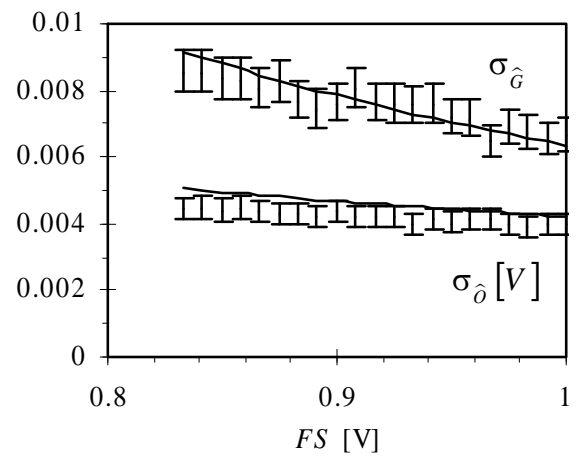

Fig. 6 - Representation of the estimated gain and offset error standard deviations as a function of the ADC Full Scale Range. We considered $n_{b}=8, M=1000, A=1.2 \mathrm{~V}, \sigma_{n}=0.1, f=200 \mathrm{~Hz}, f_{s}=200 \mathrm{kHz}$ and $N=1000$. The solid lines represent the approximation given by

(53) and (61), and the vertical bars represent the experimental results.

It can be seen, by observation of the previous figures, that the experimental results, represented by vertical bars, are below the value given by the approximate expressions (53) and (61). This validates the derivations presented here and justifies the use of those expressions to determine an upper bound for estimated ADC gain and offset error.

\section{CONCLUSIONS}

In this paper we analyzed the precision of the estimates of the independently based ADC gain and offset error obtained with the Standard Histogram Method. The main results were expressions (53) and (61) that can be used to determine the expanded uncertainty, and corresponding uncertainty interval, for the estimated independently based gain and offset error. This has the same importance as in any measurement system where the quality of the measurements should be expressed in terms of confidence intervals for the results. 


\section{ACKNOWLEDGMENTS}

This work was sponsored by the Portuguese national research project entitled "InADC - Estudo Detalhado de Métodos de Ensaio de Conversores Analógico-Digitais”, reference PTDC/EEA-ELC/ $68843 / 2006$ and project "Influence of phase noise and jitter in the testing of telecommunication ADCs", reference IT/LA/295/2005, whose support the authors gratefully acknowledge.

\section{REFERENCES}

[1] J. Blair, "Histogram measurement of ADC non linearities using sine waves", IEEE Transactions on Instrumentation and Measurement, vol. 43, n 3, pp. 373-383, June 1994.

[2] Paolo Carbone and Dario Petri, "Noise Sensitivity of the ADC Histogram Test”, IEEE Transactions on Instrumentation and Measurement, vol. 473, n 4, pp. 1001-1004, June 1998.

[3] G. Chiorboli and C. Morandi, "About the Number of Records to be Acquired for Histogram Testing of A/D Converters using Synchronous Sinewave and Clock Generators”, in Proceedings of the 4th Workshop on ADC Modeling and Testing, Bordeaux, France, September 9-10, 1999, pp. 182-186.

[4] Paolo Carbone and Giovanni Chiorboli, "ADC Sinewave Histogram Testing with Quasi-choerent Sampling”, Proceedings of the 17th IEEE IMTC Conference, vol. 1, pp. 108-113, May 2000, Baltimore, USA.

[5] F. Corrêa Alegria and A. Cruz Serra, "Influence of Frequency Errors in the Variance of the Cumulative Histogram”, IEEE Transactions on Instrumentation and Measurements, vol. 50, n. 2, April 2001, pp. 461-464.

[6] F. Corrêa Alegria and A. Cruz Serra, "Study of the Variance in the Histogram test of ADCs", Proc. of ConfTele 2001, Figueira da Foz, Portugal, April 23-24, 2001, pp. 141-145.

[7] F. Corrêa Alegria and A. Cruz Serra, "Variance of the Cumulative Histogram of ADCs due to Frequency Errors", Proc. of the IEEE Instrumentation and Measurement Technology Conference, Budapeste, Hungaria, May 21-23, 2001, pp. 2021-2026.

[8] F. Corrêa Alegria and A. Cruz Serra, "Uncertainty in the ADC Transition Voltages Determined with the Histogram Method", Proc. of the 6th Workshop on ADC Modeling and Testing, Lisboa, Portugal, September 13-14, 2001, pp. 28-32.

[9] F. Corrêa Alegria and A. Cruz Serra, "Variance of the Cumulative Histogram of ADCs due to Frequency Errors", IEEE Transactions on Instrumentation and Measurements, vol. 52, n. ${ }^{\circ}$ 1, February 2003, pp. 69-74.

[10] IEEE, "IEEE Standard for digitizing waveform recorders IEEE Std 1057-1994”, Institute of Eletrical and Electronics Engineers, Inc., SH94245, December 1994.

[11] Petruccelli, Nandram and Chen, "Applied Statistics for Engineers and Scientists”, Prentice Hall, 1999.

[12] Neter, Kutner, Nachtsheim and Wasserman, "Applied Linear Statistical Models”, $4^{\text {th }}$ Edition, Irwin, 1996.

[13] F. Corrêa Alegria and A. Cruz Serra, "Error in the estimation of transition voltages with the standard histogram test of ADCs”, Measurement, Elsevier Science, June 2004, vol. 35, n. 4, pp. 389-397.

[14] Sheldon Ross, “A First Course in Probability”, 6 ${ }^{\text {th }}$ Edition, Prentice Hall, 2001.

[15] Athanasios Papoulis, "Probability, Random Variables and Stochastic Processes”, McGraw-Hill, $3^{\text {rd }}$ edition, 1991. 